\title{
Hemoadsorption for management of Patients on Veno-venous ECMO Support for Severe COVID-19 Acute Respiratory Distress Syndrome
}

Travis Geraci ${ }^{1}$, Zachary Kon ${ }^{2}$, Nader Moazami ${ }^{2}$, Stephanie Chang ${ }^{3}$, Julius Carillo ${ }^{2}$, Stacey $\mathrm{Chen}^{2}$, Anthony Fargnoli ${ }^{3}$, Marjan Alimi ${ }^{3}$, Harvey $\mathrm{Pass}^{3}$, Aubrey Galloway ${ }^{2}$, and Deane Smith $^{2}$

${ }^{1}$ New York University Medical Center

${ }^{2} \mathrm{NYU}$ Langone Health

${ }^{3}$ Affiliation not available

April 20, 2021

\begin{abstract}
Background and aim of the study: Patients with severe coronavirus disease 2019 (COVID-19) develop a profound cytokinemediated pro-inflammatory response. This study reports outcomes in 10 patients with COVID-19 supported on veno-venous extracorporeal membrane oxygenation (VV-ECMO) who were selected for the emergency use of a hemoadsorption column integrated in the ECMO circuit. Materials and Methods: Pre and post treatment, clinical data and inflammatory markers were assessed to determine the safety and feasibility of using this system, and to evaluate the clinical effect. Results: During hemoadsorption, median levels of interleukin (IL)-2R, IL-6, and IL-10 decreased by 54\%, 86\%, and $64 \%$ respectively. Reductions in other markers were observed for LDH (-49\%), ferritin (-46\%), D-dimer (-7\%), C-reactive protein (-55\%), procalcitonin (-76\%) and lactate $(-44 \%)$. Vasoactive-inotrope scores decreased significantly over the treatment interval $(-80 \%)$. The median hospital length of stay was 53 days (36-85) and at 90-days post cannulation, survival was $90 \%$ which was similar to a group of patients without the use of hemoadsorption. Conclusions: Addition of hemoadsorption to VV-ECMO in patients with severe COVID-19 is feasible and reduces measured cytokine levels. However, in this small series, the precise impact on the overall clinical course and survival benefit still remains unknown.
\end{abstract}

Title: Hemoadsorption for management of Patients on Veno-venous ECMO Support for Severe COVID-19 Acute Respiratory Distress Syndrome

Running Title: Hemoadsoprtion for COVID-19

Authors:

Travis C Geraci, MD

New York University Langone Health

Department of Cardiothoracic Surgery

Travis.geraci@nyulangone.org

Zachary N Kon, MD

New York University Langone Health

Department of Cardiothoracic Surgery 
Zachary.kon@nyulangone.org

Nader Moazami MD

New York University Langone Health

Department of Cardiothoracic Surgery

Nader.moazami@nyulangone.org

Stephanie H Chang MD

New York University Langone Health

Department of Cardiothoracic Surgery

Stephanie.chang@nyulangone.org

Julius Carillo, MD

New York University Langone Health

Department of Cardiothoracic Surgery

Julius.carillo@nyulangone.org

Stacey Chen, MD

New York University Langone Health

Department of Cardiothoracic Surgery

Stacey.chen@nyulangone.org

Anthony Fargnoli, PhD

New York University Langone Health

Department of Cardiothoracic Surgery

Anthony.fargnoli@nyulangone.org

Marjan Alimi, MD

New York University Langone Health

Department of Cardiothoracic Surgery

Marjan.alimi@nyulangone.org

Harvey Pass, MD

New York University Langone Health

Department of Cardiothoracic Surgery

Harvey.pass@nyulangone.org

Aubrey Galloway MD

New York University Langone Health

Department of Cardiothoracic Surgery

Aubrey.galloway@nyulangone.org

Deane E Smith, MD 
New York University Langone Health

Department of Cardiothoracic Surgery

Deane.smith@nyulangone.org

Address correspondence to :

Travis C Geraci, MD

Assistant Professor of Cardiothoracic Surgery

New York University Langone Health

Department of Cardiothoracic Surgery

$5301^{\text {st }}$ Avenue, Suite 9V, New York, NY 10016

Tel: 212-263-8134

Email: travis.geraci@nyulangone.org

Disclosures/ Conflict of Interest Statement: Dr. Geraci, Dr. Smith, Dr. Chen, Dr. Chang, Dr. Fargnoli, Dr. Carillo, and Dr. Alimi have no disclosures. Dr. Kon discloses a financial relationship with Medtronic, Inc and Breethe, Inc.; Dr. Moazami with Medtronic, Inc,; Dr. Pass obtained a grant from NYU Langone Health for COVID-related research; and Dr. Galloway with Edward Lifesciences and Medtronic, Inc.

Funding Statement: There was no source of funding for this manuscript.

Data availability statement: Data included in this manuscript is available for review upon reasonable request.

\section{Abstract}

Background and aim of the study: Patients with severe coronavirus disease 2019 (COVID-19) develop a profound cytokine-mediated pro-inflammatory response. This study reports outcomes in 10 patients with COVID-19 supported on veno-venous extracorporeal membrane oxygenation (VV-ECMO) who were selected for the emergency use of a hemoadsorption column integrated in the ECMO circuit.

Materials and Methods: Pre and post treatment, clinical data and inflammatory markers were assessed to determine the safety and feasibility of using this system, and to evaluate the clinical effect.

Results: During hemoadsorption, median levels of interleukin (IL)-2R, IL-6, and IL-10 decreased by 54\%, $86 \%$, and $64 \%$ respectively. Reductions in other markers were observed for LDH $(-49 \%)$, ferritin $(-46 \%)$, D-dimer $(-7 \%)$, C-reactive protein $(-55 \%)$, procalcitonin $(-76 \%)$ and lactate $(-44 \%)$. Vasoactive-inotrope scores decreased significantly over the treatment interval $(-80 \%)$. The median hospital length of stay was 53 days (36-85) and at 90 -days post cannulation, survival was $90 \%$ which was similar to a group of patients without the use of hemoadsorption.

Conclusions: Addition of hemoadsorption to VV-ECMO in patients with severe COVID-19 is feasible and reduces measured cytokine levels. However, in this small series, the precise impact on the overall clinical course and survival benefit still remains unknown.

\section{Abbreviations}

COVID-19, coronavirus disease 2019

VV-ECMO, veno-venous extracorporeal membrane oxygenation

FDA, Food and Drug Administration

IL, interleukin 
ARDS, acute respiratory distress syndrome

$\mathrm{PaO}_{2}$, partial pressure of oxygen

$\mathrm{FiO}_{2}$, fraction of inspired oxygen

$\mathrm{P} / \mathrm{F}$ ratio, partial pressure of oxygen to fraction of inspired oxygen ratio

$\mathrm{PCO}_{2}$, partial pressure of carbon dioxide

PTT, partial thromboplastin time

PAMP, pathogen-associated molecular patterns

DAMP, damage-associated molecular patterns

BMI, body mass index

LDH, lactate dehydrogenase

HIT, heparin-induced thrombocytopenia

\section{Introduction}

Patients with severe coronavirus disease 2019 (COVID-19) often develop a systemic inflammatory response syndrome (SIRS) that may result in hemodynamic instability and multisystem organ failure. ${ }^{1}$ Elevated levels of inflammatory cytokines are reported in the peripheral blood and lung tissue of critically ill patients with COVID-19, which correlate with disease severity and mortality. ${ }^{2}$ Mechanical ventilation is required in approximately $20 \%$ of patients admitted with COVID-19 for progressive hypoxic or hypercarbic respiratory failure and is associated with a mortality rate of $24-74 \% .^{3,4}$ Veno-venous extracorporeal membrane oxygenation (VV-ECMO) has been employed in select patients with COVID-19 failing mechanical ventilation. $., 6,7,8$ Once supported on ECMO, there are limited effective therapeutic options targeting the dysregulated immune response in patients with severe COVID-19. ${ }^{9}$ We previously reported excellent survival with this modality in a subset of patients who required VV-ECMO at our institution. ${ }^{5}$

Experience from prior hyperinflammatory syndromes suggest that reducing levels of circulating cytokines and pro-inflammatory mediators may help attenuate an excessive immune response, limiting further tissue injury and leading to clinical recovery. ${ }^{10,11}$ Three types of blood purification modalities are available, including filtration, dialysis, and adsorption. International registries have been created to evaluate the feasibility and safety of hemoadsorption devices for patients with COVID-19, including the oXiris membrane (Baxter, Meyzieu, France), the Spectra Optia Apheresis System (Terumo BCT Inc, Lakewood, CO, USA), and CytoSorb $^{\mathrm{TM}}$ (CytoSorbents, Inc. Berlin, Germany). ${ }^{12}$

CytoSorb is an extracorporeal polystyrene-based hemoadsorption device designed to filter blood inflammatory mediators based on molecular size and concentration, including cytokines, chemokines, and bacterial exotoxins. ${ }^{9}$ In early 2020, it received a Food and Drug Administration (FDA) emergency use authorization for integration into bypass, ECMO, or dialysis circuits in patients with COVID-19. Some clinical benefits, including clearance of lactate, reduced vasopressor requirement and improved survival, have been reported in case series for patients with acute respiratory distress syndrome (ARDS) and in patients with uncontrolled septic shock. ${ }^{13-15}$ In patients with SIRS following prolonged cardiopulmonary bypass and in patients undergoing surgery for infective endocarditis, the use of hemoadsorption is associated with hemodynamic stabilization and reversal of organ dysfunction. ${ }^{16,17}$ In addition, this device has been shown to be compatible with ECMO without an increase in adverse events. ${ }^{18,19}$

The therapeutic consequence of active reduction of inflammatory cytokines and mediators in patients with severe COVID-19 is unknown. During the COVID-19 pandemic, we obtained emergency use authorization for CytoSorb in patients with severe COVID-19 and respiratory failure requiring veno-venous extracorporeal membrane oxygenation (VV-ECMO). This study reports inflammatory and clinical outcomes in patients with severe COVID-19 treated at our institution. The primary aim was to assess the safety and feasibility 
of hemoadsorption in this population. Secondarily, we assessed the anti-inflammatory efficacy of adsorption by comparison of pre and post treatment levels of cytokines, inflammatory markers, and clinical parameters.

\section{Materials and Methods}

A single institution retrospective analysis was conducted of patients with severe COVID-19 and respiratory failure placed on VV-ECMO, with the addition of hemoadsorption at New York University Langone Health from March 10, 2020 to June 30, 2020. Cytosorb adsorption is not currently approved by the United States Food and Drug Administration. In the midst of the COVID-19 pandemic, however, we obtained emergency use authorization for select patients. The primary objective of this study was to determine the safety and feasibility of hemoadsorption therapy based on clinical outcomes, including adverse events and survival. Secondarily, pre and post treatment levels of select cytokines, biochemical data, and inflammatory markers were assessed to determine treatment effect. All data was collected in a prospective database and demographic, clinical, and outcomes data were collected retrospectively from the electronic medical record. Follow-up was performed until hospital discharge, or 90 days post cannulation, whichever occurred first. Study design, emergency use authorization, and data management were approved by the Institutional Review Board (IRB) at New York University Langone Health (IRB \#S20-01189). Additionally, IRB approval was granted for informed consent to be obtained from family members and/or health care decision-makers on the patient's behalf given the severity of illness and need for sedation.

\section{Patient Selection and Management}

The diagnosis of COVID-19 was established by nasal pharyngeal swab for reverse transcriptase polymerase chain reaction assay. Patients were evaluated, cannulated for VV-ECMO, and managed by a multidisciplinary team of cardiothoracic surgeons and critical care physicians. ${ }^{5}$ Entry and inclusion criteria were based on the arterial partial pressure of oxygen $\left(\mathrm{PaO}_{2}\right)$-to-fraction of inspired oxygen $\left(\mathrm{FiO}_{2}\right)$ ratio $(\mathrm{P} / \mathrm{F}$ ratio $)$, arterial blood gas, ventilator settings, and patient functional status, comorbidities, and hemodynamic status. ECMO support was only offered to patients with a $\mathrm{P} / \mathrm{F}$ ratio of less than $150 \mathrm{~mm} \mathrm{Hg}$ or a $\mathrm{pH}$ of less than 7.25 with a partial pressure of arterial carbon dioxide $\left(\mathrm{PaCO}_{2}\right)$ exceeding $60 \mathrm{~mm} \mathrm{Hg.}{ }^{5} \mathrm{~A}$ lung-protective ventilation strategy was employed with titration of ECMO circuit flow, which was maintained above $3 \mathrm{~L} / \mathrm{min}$ to limit oxygenator thrombosis. Oxygenator $\mathrm{FiO}_{2}$ was maintained at 1.0 for the entirety of support. The sweep gas flow rate was adjusted to achieve a goal partial pressure of carbon dioxide $\left(\mathrm{PCO}_{2}\right)<45 \mathrm{mmHg}$. Anticoagulation using intravenous heparin infusion was used to achieve a partial thromboplastin time (PTT) goal of 50-70 and/or anti-Xa level $>0.15$ units/mL based on prior experience. ${ }^{11,12,20}$ Vasopressors were used as needed to maintain a mean arterial pressure goal of $60 \mathrm{mmHg}$.

The ECMO multidisciplinary team also evaluated patients for hemoadsorption. Hemoadsorption was added to the circuit in selected patients based on severity of illness - those who had progressive clinical decline while supported on VV-ECMO - including assessment of the arterial partial pressure of oxygen $\left(\mathrm{PaO}_{2}\right)$ to fraction of inspired oxygen $\left(\mathrm{FiO}_{2}\right)$ ratio $(\mathrm{P} / \mathrm{F}$ ratio), arterial blood gas values, ventilator and ECMO settings, and hemodynamic status, particularly if those requiring high dose vasopressors. Patients with thrombocytopenia $<20,000 / \mu \mathrm{L}$ or with a history of heparin-induced thrombocytopenia were excluded per established contraindications to use. There were no other absolute exclusion criteria.

\section{Hemoadsorbption}

In select patients on VV-ECMO, a hemoadsorption column (300 $\mathrm{mL}$ device) was installed in parallel with the ECMO circuit in a pre-hemofilter position (Figure 1). Filtered blood returned to the venous line prior to the centrifugal pump. An additional ultrasonic flow probe was placed to calculate flow through the cartridge. All device integrations and exchanges required a brief suspension of ECMO. Treatment was planned for a 72-hour duration with device exchange every 12 hours for the first 24 hours, then 24 hours thereafter, or as needed in the event of cartridge thrombosis.

\section{Statistical Analysis}

Descriptive analyses for categorical variables are reported in Table 1 and Table 3 as frequency and percentage. 
Continuous variables are reported as means and standard deviations or errors for normally distributed variables and as either means and range or medians and interquartile ranges for non-normally distributed variables. Single one-way analysis of variance (ANOVA) test was performed for inflammatory and cytokine markers for data compiled per group time points of (-1 day prior to treatment), during the course (24 hours, 48 hours, 72 hours), and Post (2-3 days after removal) to assess changes in overall profile. Inflammatory, metabolic, and clinical data reported in Table 3 evaluated differences in the mean changes per patient as defined as each corresponding patient's change in status over time as: $\%$ Change $=$ (Post treatment Pre treatment)/(Pre treatment), with negative values indicating a decrease. A p-value less than 0.05 was predetermined to be statistically significant. All statistical analyses were performed with $\mathrm{R}$ software, version 4.0.2 (R Foundation for Statistical Computing) and SPSS.

\section{Results}

From March 10, 2020 to June 30, 2020, 10 patients with respiratory failure from severe COVID-19 underwent VV-ECMO cannulation with the addition of hemoadsportion. Median age was 45 (IQR 37-51), predominantly male (90\%), with a median body mass index (BMI) of $33 \mathrm{~kg} / \mathrm{m}^{2}$ (IQR 30-37) and the majority had no diagnosed prior medical history besides obesity. At the time of VV-ECMO cannulation, patients selected for hemoadsorption had a median $\mathrm{pH}$ of 7.24 (IQR 7.07-7.41), $\left(\mathrm{PCO}_{2}\right)$ of 63 (IQR 41-86) and a P:F ratio of 85. Prior to cannulation, 7 patients $(70 \%)$ required vasopressor and/or inotropic hemodynamic support with a median vasoactive inotrope score was 4.79. Patients selected for hemoadsorption were hospitalized for a median of 4.5 days (IQR 1.8-11.5) prior to ECMO and were supported on EMCO a median of 1 day (IQR 0-3) prior to the initiation of treatment. Baseline demographic information and clinical characteristics are reported in Table 1.

\section{Hemoadsorption}

Of the 10 patients selected for hemoadsorption, 8 patients completed a full 72-hour treatment duration, and there were no complications or adverse events in 8 patients (Table 2). One patient was removed from treatment after 24 hours due to an inability to maintain adequate paralysis and sedation, which subsequently improved with cessation of hemoadsorption. Another patient developed heparin-induced thrombocytopenia (HIT) and the device was removed after 60 hours of treatment. There was no evidence of progressive end-organ injury attributed to therapy with reductions in median levels of pre and post-hemoadsorption in creatinine (-7\%) and aspartate aminotransferase/ alanine aminotransferase levels (-10\%/ $-40 \%)$.

Cytokines, Inflammatory, and Biochemical Data

After the planned 72-hour treatment course, patients who underwent hemoadsorption, had a median decrease in levels of interleukin (IL) 2 Receptor (IL-2R), IL-6, and IL-10 by $54 \%, 86 \%$, and $64 \%$ respectively (Figure $2)$. Reductions in LDH (-49\%), ferritin (-46\%), D-dimer (-7\%), C-reactive protein (-55\%), procalcitonin ($76 \%$ ) and lactate (-44\%) were also observed (Figure 3). All pre and post-treatment cytokine, inflammatory, and biochemical data are reported in Table 3.

\section{Clinical Outcomes}

Overall survival of patients who underwent hemoadsorption on VV-ECMO was 90\% (9/10 patients) (Table 4) with a median length of stay of 47 days (IQR 34-88) and a median of 22 days (IQR 13-64) on VV-ECMO. Lung recovery, defined as the interval between intubation until the patient was on room air for 24 hours, was achieved in median 34 days (IQR 26-51) in patients who underwent hemoadsorption. A single patient who underwent CytoSorb remained admitted at 90-days post ECMO cannulation with ongoing respiratory failure requiring mechanical ventilation. Although this is not a comparative study, for context, 90-day survival was also $90 \%$ in patients on VV-ECMO who did not undergo hemoadsorption therapy.

\section{Discussion}

In a single institution study of patients with severe COVID-19 and respiratory failure placed on VV-ECMO, 10 patients were selected for the addition of hemoadsorption to the circuit. Our data support the feasibility 
of this treatment for patients with COVID-19 on VV-ECMO with limited adverse events. During the treatment interval, patients had reductions in circulating cytokines and reductions in clinically relevant inflammatory markers. Equally, patients rapidly cleared their vasopressor requirement. Given the limited sample size and heterogeneous clinical course of these complex patients with severe COVID-19, the overall clinical contribution of hemoadsorption towards pulmonary recovery and overall survival, remains unknown despite the favorable trends observed in reduction of inflammatory profile.

There were limited adverse events associated with integration of the column and eight patients (80\%) completed a full 72-hour treatment duration (Table 2). In the setting of full heparin anticoagulation in all patients, thrombocytopenia was observed in the majority of patients $(60 \%)$. This reduction, however, did not result in bleeding events. Previous series of critically ill patients undergoing hemoadsorption have reported modest reductions in platelet count, which were typically mild $(<10 \%)$ and self-limiting. ${ }^{22}$ In our series, one patient developed heparin induced thrombocytopenia (HIT) and was removed from treatment after 60 hours. It was unclear, however, whether the device was the precipitating factor for HIT. In our previously published series, 4/17 patients (24\%) with severe COVID-19 on VV-ECMO without hemoadsorption, tested positive for HIT by platelet factor 4 antibody assay, two of which were confirmed positive by serotonin release assay. Given these findings, nonetheless, close monitoring for thrombocytopenia and HIT during hemoadsorption is warranted. Additionally, in 3 patients, the device required unplanned exchange for cartridge thrombosis, all of which were of low clinical impact.

Three patients required increased dosing of sedatives and neuromuscular blockade agents, including one patient who was removed from treatment after 24 hours due to an inability to maintain adequate paralysis and sedation. A number of in-vitro and in-vivo studies have shown reduced or eliminated levels of analgesics, sedatives, and antibiotics during hemoadsorption. ${ }^{23}$ Given the multifaceted pharmacologic regimens of patients with severe COVID-19, it is critical to consistently asses the patient's physical exam and monitor drug levels to assure adequate therapeutic delivery during hemoadsorption.

Elevated levels of cytokines and inflammatory mediators in patients with COVID-19 correlate with disease severity, supporting the notion of an uncontrolled cytokine storm. ${ }^{2}$ Patients receiving hemoadsorption had significant decreases in pro-inflammatory (IL-2R by $54 \%$; and IL-6 by $86 \%$ ) and anti-inflammatory (IL10 by $64 \%$ ) cytokines. International registry data have shown similar significant reductions of IL-6 with hemoadsorption in a heterogeneous population of critically ill patients. ${ }^{24}$ Equally, patients treated with hemoadsorption had reductions in LDH (-49\%), ferritin (-46\%), D-dimer (-7\%), C-reactive protein (-55\%), procalcitonin $(-76 \%)$ and lactate $(-44 \%)$.

The clinical consequence of the clearance of inflammatory mediators remains unanswered; the number of patients in this series is small and the study is underpowered to detect subtle differences. Given that the use of hemoadsorption was through an emergency use authorization in the context of an evolving pandemic, we were not able to perform a comparative trial of patients on ECMO with and without hemoadsorption. There were, however, some notable clinical outcomes observed. Of the 7 patients requiring vasopressor and/or inotropic support pre-CytoSorb, only 2 required hemodynamic support afterinitiation of hemoadsorption and these levels were significantly reduced. The pre and post-treatment median vasopressin inotropic scores decreased by $80 \%$ during the 3-day treatment interval. In patients who underwent VV-ECMO with CytosSorb, median hospital length of stay was 53 days (36-85) and survival was $90 \%$ with 8 patients discharged and 1 patient hospitalized at 90-days post ECMO cannulation. This patient was discharged to an acute rehabilitation facility after a 160 day length of stay and discharged home after 33 days of rehabilitation.

The survival rate in this series is substantially higher than other institutional reports of patients on VVECMO for COVID-19, with survival to discharge rates between $56.8-62 \%{ }^{7,8}$ In this small study, the causal contribution of hemoadsorption towards respiratory recovery and survival is unproven. Furthermore, in the patients on hemoadsorption, the column was removed after 72 hours of usage. It is unclear if longer duration of support would have contributed to clinical recovery. This study is further limited by its retrospective, single-center design with limited sample size, and is therefore underpowered for definitive lung recovery or survival analysis. Patients were selected for the addition of hemoadsorption in a non-random fashion based 
on severity of illness. There was an inherent selection bias, therefore, for patients with more severe disease, given the lack of clear inclusion or exclusion criteria. Given the limited therapeutic options available for severely ill patients supported on VV-ECMO, however, we instituted the compassionate use of hemoadsorptionvia emergency use authorization. Furthermore, the results are confounded by the multitude of additional treatments and interventions that were used to treat these complex patients with COVID-19. Ultimately, randomized trials with defined treatment algorithms will be required to establish definable clinical benefits of hemoadsorption in patients with severe COVID-19.

\section{Conclusions}

Use of hemoadsorption with VV-ECMO in patients with severe COVID-19 respiratory failure is feasible with limited adverse events. Hemoadsorption was associated with thrombocytopenia and filtration of certain medications including sedatives and neuromuscular blockade agents, which warrants close clinically monitoring. In this pilot study, patients on hemoadsorption had greater reductions in clinically relevant inflammatory markers, lactate, and reduction in vasopressor requirements. Positive contribution to clinical course and survival remains unclear Additional research is necessary to define the role of hemoadsorption in patients with severe COVID-19.

\section{Authors Contributions:}

- Travis C Geraci, MD: Data analysis/ interpretation, drafting article, critical revision of article, data collection, statistics.

- Zachary N Kon, MD: Concept/design, data analysis/ interpretation, critical revision of article.

- Nader Moazami MD: Drafting article, concept/design, data analysis/ interpretation, critical revision of article.

- Stephanie H Chang MD: Concept/design, data collection.

- Julius Carillo, MD: Concept/design, data collection.

- Stacey Chen, MD: Concept/design, data collection, statistics.

- Anthony Fargnoli, PhD: Concept/design, data collection, statistics.

- Marjan Alimi, MD: Concept/design, data collection, statistics.

- Harvey Pass, MD: Concept/design, data collection, statistics.

- Aubrey Galloway MD: Concept/design, data analysis/ interpretation, critical revision of article.

- Deane E Smith, MD: Concept/design, data analysis/ interpretation, critical revision of article.

\section{References}

1. Ye Q, Wang B, Mao J. The pathogenesis and treatment of the 'Cytokine Storm' in COVID-19. J Infect. 2020;80(6):607-613.

2. Huang C, Wang Y, Li X, et al. Clinical features of patients infected with 2019 novel coronavirus in Wuhan, China. Lancet. 2020 Feb 15;395(10223):497-506.

3. Richardson S, Hirsch JS, Narasimhan M, et al. Presenting Characteristics, Comorbidities, and Outcomes Among 5700 Patients Hospitalized With COVID-19 in the New York City Area. JAMA. 2020 May 26;323(20):2052-2059.

4. Cummings MJ, Baldwin MR, Abrams D, et al. Epidemiology, clinical course, and outcomes of critically ill adults with COVID-19 in New York City: a prospective cohort study. Lancet. 2020;395(10239):17631770.

5. Kon ZN, Smith DE, Chang SH, et al. Extracorporeal Membrane Oxygenation Support in Severe COVID-19. Ann Thorac Surg. 2020;S0003-4975(20)31152-8.

6. Mustafa AK, Alexander PJ, Joshi DJ, et al. Extracorporeal Membrane Oxygenation for Patients With COVID-19 in Severe Respiratory Failure. JAMA Surg. 2020 Aug 11;155(10):990-2.

7. Barbaro RP, MacLaren G, Boonstra PS, et al. Extracorporeal Life Support Organization. Extracorporeal membrane oxygenation support in COVID-19: an international cohort study of the Extracorporeal Life Support Organization registry. Lancet. 2020 Oct 10;396(10257):1071-1078.

8. Shih E, DiMaio JM, Squiers JJ, et al. Venovenous extracorporeal membrane oxygenation for patients 
with refractory coronavirus disease 2019 (COVID-19): Multicenter experience of referral hospitals in a large health care system. J Thorac Cardiovasc Surg. 2020 Dec 1:S0022-5223(20)33145-7.

9. Soy M, Keser G, Atagündüz P, et al. Cytokine storm in COVID-19: pathogenesis and overview of anti-inflammatory agents used in treatment. Clin Rheumatol. 2020;39(7):2085-2094.

10. Napp LC, Ziegeler S, Kindgen-Milles D. Rationale of Hemoadsorption during Extracorporeal Membrane Oxygenation Support. Blood Purif. 2019;48(3):203-214.

11. Lother A, Benk C, Staudacher DL, et al. Cytokine Adsorption in Critically Ill Patients Requiring ECMO Support. Front Cardiovasc Med. 2019;6:71.

12. Swol J, Lorusso R. Additive treatment considerations in COVID-19-The clinician's perspective on extracorporeal adjunctive purification techniques. Artif Organs. 2020 Sep;44(9):918-925.

13. Mitzner SR, Gloger M, Henschel J, et al. Improvement of hemodynamic and inflammatory parameters by combined hemoadsorption and hemodiafiltration in septic shock: a case report. Blood Purif. 2013;35(4):314-315.

14. Akil A, Ziegeler S, Reichelt J, et al. Combined Use of CytoSorb and ECMO in Patients with Severe Pneumogenic Sepsis [published online ahead of print, 2020 Apr 6]. Thorac Cardiovasc Surg. 2020;10.1055/s-0040-1708479.

15. Kogelmann K, Jarczak D, Scheller M, et al. Hemoadsorption by CytoSorb in septic patients: a case series. Crit Care. 2017;21(1):74.

16. Träger K, Fritzler D, Fischer G, et al. Treatment of post-cardiopulmonary bypass SIRS by hemoadsorption: a case series. Int J Artif Organs. 2016;39(3):141-146.

17. Träger K, Skrabal C, Fischer G, et al. Hemoadsorption treatment of patients with acute infective endocarditis during surgery with cardiopulmonary bypass - a case series. Int J Artif Organs. 2017;40(5):240249.

18. Kogelmann K, Scheller M, Drüner M, et al. Use of hemoadsorption in sepsis-associated ECMOdependent severe ARDS: A case series. J Intensive Care Soc. 2020;21(2):183-190.

19. Bonavia A, Groff A, Karamchandani K, Singbartl K. Clinical Utility of Extracorporeal Cytokine Hemoadsorption Therapy: A Literature Review. Blood Purif. 2018;46((4)):337-49.

20. CytoSorb, Manufacture Guideline, CytoSorb $300 \mathrm{~mL}$ Device: Approved by FDA for Emergency Treatment of COVID-19. https://cytosorb-therapy.com/en-us/. Accessed, 10/1/200.

21. Gruda MC, Ruggeberg KG, O'Sullivan P, et al. Broad adsorption of sepsis-related PAMP and DAMP

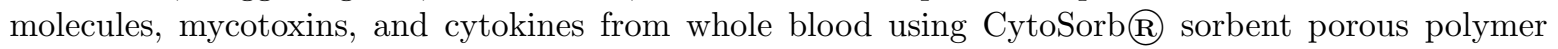
beads. PLoS One. 2018;13(1):e0191676.

22. Ankawi G, Xie Y, Yang B, et al. What Have We Learned about the Use of Cytosorb Adsorption Columns? Blood Purif. 2019;48(3):196-202.

23. Zoller M, Döbbeler G, Maier B, Vogeser M, Frey L, Zander J. Can cytokine adsorber treatment affect antibiotic concentrations? A case report. J Antimicrob Chemother. 2015 Jul;70((7)):2169-71.

24. Friesecke S, Träger K, Schittek GA, et al. International registry on the use of the CytoSorb adsorber in ICU patients: Study protocol and preliminary results. Med Klin Intensivmed Notfmed. 2019;114(8):699707.

Table 1: Clinical and Demographic Characteristics of Patients with COVID-19 on VV- ECMO with or without CytoSorb

\section{Variable}

Age, median (IQR), years

Sex, No (\%) Male Female

Race, No (\%) Latino/ Hispanic White/ Caucasian Asian/ Asian-American Black/ African American Other/ not-specified BMI, $\mathrm{kg} / \mathrm{m}^{2}$, median (IQR)

Smoking history, No (\%) Never Smoker Current smoker Former smoker Not assessed/ unknown

Comorbid conditions, No (\%) None Obesity (BMI > 30) Hyperlipidemia Hypertension Diabetes mellitus Chronic respirator Interventions prior to ECMO Prone positioning Neuromuscular blockade Inhaled nitric oxide Continuous renal replacement At time of VV-ECMO cannulation, median (IQR) Arterial blood gas pH PO2 PCO2 PaO2/FiO2 (P:F ratio) Mechanical ve 
Hospital days prior to mechanical ventilation, median (IQR)

Hospital days with mechanical ventilation prior to VV-ECMO cannulation, median (IQR)

Hospital days prior to VV-ECMO cannulation, median (IQR)

Days on VV-ECMO prior to CytoSorb, median (IQR)

Abbreviations: VV-ECMO, veno-venous extracorporeal membrane oxygenation; IQR, interquartile range; BMI, body mass index

Table 2: Adverse Events during CytoSorb Treatment Period

\begin{tabular}{|c|c|c|c|c|c|}
\hline Patient & $\begin{array}{l}\text { Change Out } \\
\# \mathbf{1} 12 \text { Hours }\end{array}$ & $\begin{array}{l}\text { Change Out } \\
\# 212 \text { Hours }\end{array}$ & $\begin{array}{l}\text { Change Out } \\
\# \mathbf{3} 24 \text { Hours }\end{array}$ & $\begin{array}{l}\text { Change Out } \\
\# 424 \text { Hours }\end{array}$ & Adverse Event \\
\hline 1 & & & & & No events \\
\hline 2 & & & & & No events \\
\hline 3 & & & & & No events \\
\hline 4 & & & & & No events \\
\hline 5 & & & & & No events \\
\hline 6 & & $\mathrm{D} / \mathrm{C}$ & $\mathrm{D} / \mathrm{C}$ & $\mathrm{D} / \mathrm{C}$ & $\begin{array}{l}\text { Filtered } \\
\text { sedatives; } \\
\text { treatment } \\
\text { discontinued } \\
\text { after } 18 \text { hours }\end{array}$ \\
\hline 7 & & & & & No events \\
\hline 8 & & & & & No events \\
\hline 9 & & & & & No events \\
\hline 10 & & HIT & & $\mathrm{D} / \mathrm{C}$ & $\begin{array}{l}\text { Progressive } \\
\text { thrombocy- } \\
\text { topenia, HIT } \\
\text { positive; } \\
\text { treatment } \\
\text { discontinued } \\
\text { after } 60 \text { hours }\end{array}$ \\
\hline
\end{tabular}

Abbreviations: C, cartridge exchange; D/C, discontinued; HIT, heparin-induced thrombocytopenia.

Table 3: Inflammatory, Metabolic and Clinical Data after 72-hour CytoSorb Treatment, or Parallel Interval on ECMO Alone

\begin{tabular}{lll}
\hline Variable & $\begin{array}{l}\text { VV-ECMO + CytoSorb, n=10 } \\
\text { Pre }\end{array}$ & $\begin{array}{l}\text { VV-ECMO + CytoSorb, } \\
\text { Post }\end{array}$ \\
Lactate dehydrogenase, U/L, median (IQR) & $856(718-1115)$ & $425(370-644)$ \\
Ferritin, ng/mL, median (IQR) & $2647(1306-2815)$ & $1004(576-2031)$ \\
D-Dimer, ng/mL, median (IQR) & $5517(2619-9363)$ & $4188(2911-8810)$ \\
C-reactive protein, mg/dL, median (IQR) & $117(31-263)$ & $64(7.8-105)$ \\
Procalcintonin, ng/mL, median (IQR) & $1.2(0.21-3.8)$ & $0.19(0.08-15)$ \\
Lactate, mmol/L, median (IQR) & $1.60(1.32-2.55)$ & $1.35(1.08-1.53)$ \\
IL-2R, pg/mL, median (IQR) & $2143(1147-15201)$ & $1279(1097-2293)$ \\
IL-6, pg/mL, median (IQR) & $22(9-618)$ & $11(7-146)$ \\
IL-10, pg/mL, median (IQR) & $18(9.5-73)$ & $5(5-83)$ \\
Hemotocrit, \%, median (IQR) & $33(30-36)$ & $30(27-32)$
\end{tabular}


Absolute lymphocyte count, 1000/ $\mathrm{L}$, median (IQR)

Platelet count, $1000 / \mu \mathrm{L}$, median (IQR)

$5(1.5-5)$

$6(4-11)$

$215(138-262) \quad 94(35-138)$

Aspartate aminotransferase, U/L, median (IQR) 39 (26-66)

$28(21-79)$

Alanine aminotransferase, U/L, median (IQR)

$41(25-72)$

$23(20-56)$

Creatinine, mg/dL, median (IQR)

$1.1(0.8-2.3)$

$1.4(0.6-1.8)$

$\mathrm{pH}$, median (IQR)

$7.38(7.28-7.42$

$7.42(7.37-7.44)$

$\mathrm{PCO}_{2}, \mathrm{mmHg}$, median (IQR)

$42(41-51)$

$43(41-44)$

FiO2, \%, median (IQR)

$40(40-40)$

$40(40-45)$

$\mathrm{PaO} 2 / \mathrm{FiO} 2$ (P:F ratio), median (IQR)

$185(152-225)$

$185(170-260)$

Vasopressin inotropic score, median (IQR)

$4.52(0-12.25)$

$1(0-2.68)$

${ }^{*}$ Grouped \%Change reported as per individual patient as $(72 \mathrm{hrs}-$ Pre $) /$ Pre, mean of change $\mathrm{p}<0.05$

Abbreviations: IL, interleukin; $\mathrm{PCO}_{2}$, Partial pressure of carbon dioxide; FiO2, fraction of inspired oxygen;

Table 4: Clinical Outcomes and Survival

\begin{tabular}{ll}
\hline Variable & VV-ECMO + CytoSorb, N=10 \\
Intubation to room air for 24-hours, days, median (IQR) & $34(26-51)$ \\
VV-ECMO, days, median (IQR) & $22(13-64)$ \\
Total hospital length of stay, days, median (IQR) & $47(34-88)$ \\
Survival Discharged Hospitalized at 90 days & $9(90 \%) 8(80 \%) 1(10 \%)$ \\
\hline
\end{tabular}

Figure 1: Diagram of CytoSorb Incorporation into the VV-ECMO Circuit. Illustration elements used with permission from CytoSorbents.

Figure 2 : Changes in Cytokine Levels during Cytosorb Hemoadsorption on VV-EMCO, (A) IL-2R, (B) IL-6, (C) IL-10.

Figure 3: Changes in Inflammatory Makers during Cytosorb Hemoadsorption on VV-ECMO, (A) Lactate dehydrogenase, (B) Ferritin, (C) C-reactive protein, (D) D-dimer.

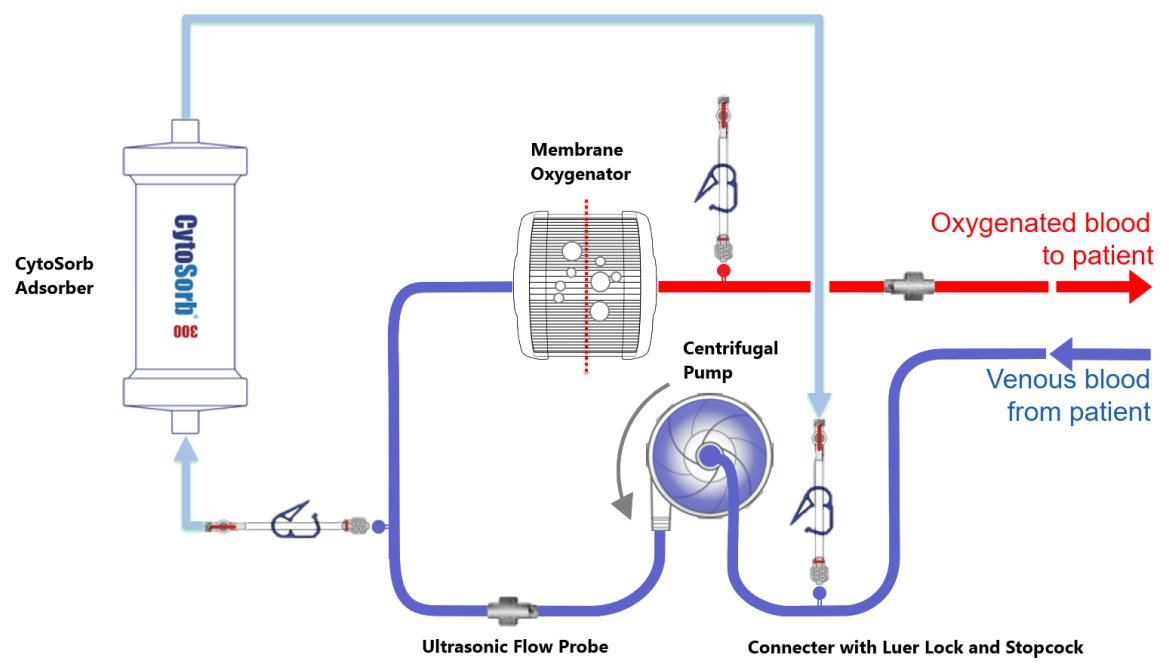



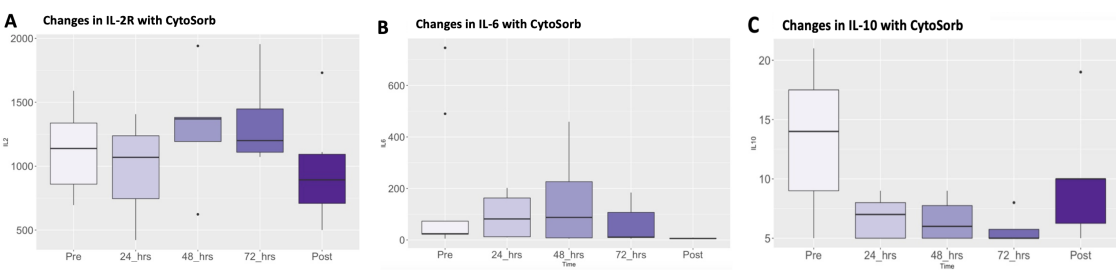

A LDH Changes: Course in CytoSorb

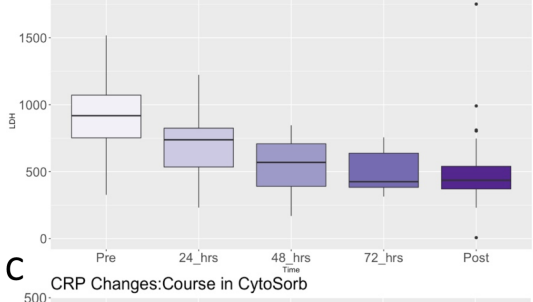

C CRP Changes:Course in CytoSorb

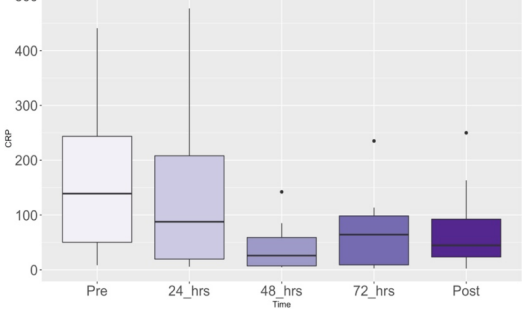

B Ferritin Changes:Course in CytoSorb

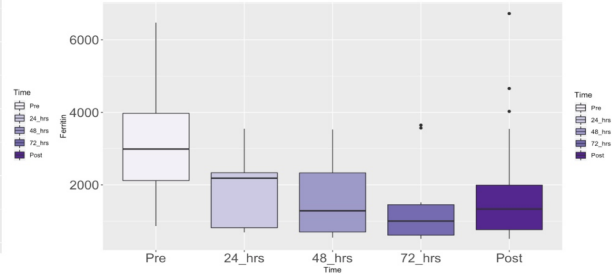

D DDIMER Changes: Course in CytoSorb

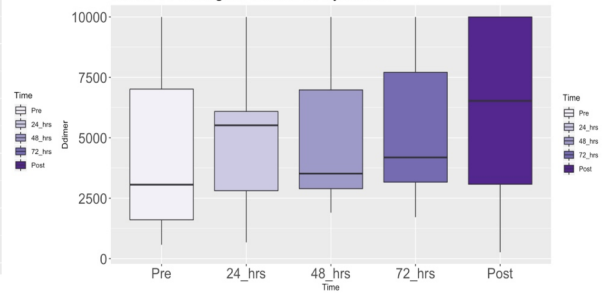

